\title{
Engineering Magnetic Nanoparticles for Thermo-Ablation and Drug Delivery in Neurological Cancers
}

\author{
Ayden Jacob ${ }^{1}$, Krishnan Chakravarthy ${ }^{2}$ \\ 1. Interventional Radiology and Oncology, UCSF School of Medicine 2. Department of Anesthesia, \\ Critical Care and Pain Medicine, University of California, San Diego
}

$\square$ Corresponding author: Krishnan Chakravarthy, kvc1008@gmail.com

Disclosures can be found in Additional Information at the end of the article

\section{Abstract}

Nanobiotechnology involves the engineering of systems, devices, and materials which can be implemented within a physiological system on a scale between 1-100nm. Nanometric materials have significant potential to exhibit unprecedented capacities for interacting with specific molecular, organellar, and cellular components of the brain via their novel optical, electronic, and structural properties. Hence, they are poised to establish new paradigms for the future of brain cancer treatment. Nanooncology is currently the most important arena in nanomedicine, and the application of nanoscale innovations in neuroscience will bring forth diagnostic and therapeutic inventions unseen hitherto in the assessment and treatment of brain cancer. Nanobiosensors, magnetic nanoparticles, and nanoparticle-chemotherapy conjugates are specific avenues within brain cancer treatment that illustrate the credible utilization of sophisticated nanobiotechnology in neurooncology. Nanotechnologically-based medical solutions are slowly progressing from the laboratory bench to clinical trials. This review aims to highlight the data that bridges the gap between laboratory benchtop and hospital bedside, as well as to illustrate potential platforms through which nanomedicine may be integrated into standardized treatment protocols against various forms of neurological cancers.

Received 03/01/2014 Review began 03/03/2014 Review ended 04/03/2014 Published 04/06/2014

C) Copyright 2014

Jacob et al. This is an open access article distributed under the terms of the Creative Commons Attribution License CC-BY 3.0., which permits unrestricted use, distribution, and reproduction in any medium, provided the original author and source are credited.
Categories: Miscellaneous, General Surgery, Oncology

Keywords: glioblastoma multiforme, nanomedicine, nanotechnology, oncology, neurooncology, drug delivery, magnetic nanoparticles, brain cancer

\section{Introduction And Background Introduction to nano-oncology}

Tumor malignancies originating in the central nervous system (CNS) account for only $2 \%$ of all cancers but are correlated with a high mortality and morbidity rate. In 2013, the National Cancer Institute reported over 23,000 new cases of CNS resident tumors and 14,080 deaths that were attributed to brain tumors [1]. Despite improvements in cancer diagnostics and therapeutics, the five-year survival rate for both localized and distant CNS tumors remains at a mere $35 \%$. Astoundingly, since 1975 , the five-year survival rate has only increased from $22.8 \%$ to $34.3 \%$ [1]. In excess of 50\% of the patients who were diagnosed with CNS tumors in 2013 were expected to die from the disease, with a median survival rate of one year [2]. Glioblastoma multiforme (GBM), a primary brain tumor, demonstrates the necessity for innovation in neurooncology treatment. As one of the greatest challenges for neurosurgery and oncology of our century, there is currently no cure for GBM, and its phenotypic symptoms are devastating. According to Jain, et al., the deficiencies in the ability to treat GBM are mostly attributed to the 


\section{Cureus}

lack of our knowledge of the pathophysiology of GBM, the limited access of chemotherapeutic agents to the site of the brain tumor, the metastatic nature of GBM, and the rapid recapitulation rate of the tumor post-surgical resection [3]. Intense research efforts toward understanding the molecular and biochemical mechanisms of brain tumors, inclusive of GBM, have provided insights into the elemental processes of these aggressive tumors, but have failed to generate novel solutions in the realms of chemotherapy, radiation treatment, or surgical procedures. Further, the utilization of imaging methods to detect the precise location and size of the tumor both pre- and post-resection is imperative in assisting clinicians with the appropriate data needed to make prognostic treatment decisions (Figure 1). Although the last century has seen little progress toward a cure for brain tumors, the rapid introduction of biotechnology into the realms of neuroscience and oncology over the last decade may provide a solution to this intractable problem.

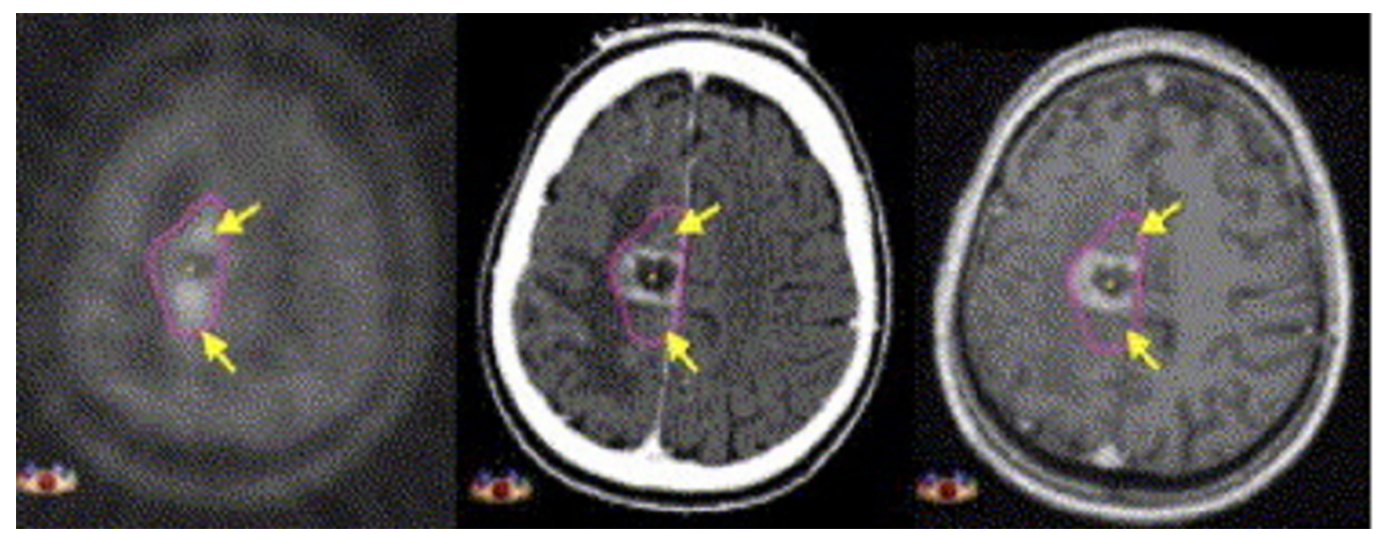

\section{FIGURE 1: Recurrent Glioblastoma Multiforme}

11C-methionine positron emission tomography shows tumor infiltration. Reused with permission from Grosu, et al. and The Intl J Rad Onc Bio Phy [4] (image license \#3334690402530).

\section{Review}

\section{Nanomedicine and neuro-oncology}

The National Nanotechnology Initiative defines nanotechnology as "the understanding and control of matter at dimensions of roughly 1-100 nm, where unique phenomena enable novel applications" [5]. Nanoscience enables researchers to study the fundamental building blocks of nature by creating materials and devices which peer through matter at the nanoscale. Gaining new insights into physiological systems at the miniscule level of atoms and molecules has given rise to the field of nanobiotechnology: the application of nanotechnology to the life sciences [6]. A result of nanobiotechnology is the development of nanomedicine, a specific field of medicine which utilizes the ability to study living cells at the nanoscale in order to implement innovative methods of diagnostics and therapeutics in medical disease states. An important facet of nanomedicine today is nanooncology, which is the application of nanobiotechnology in medicine to study, diagnose, and treat various forms of cancer. Basic brain cancer research is progressively incorporating nanobiotechnologies to translate hypothetical forms of cancer treatment into innovative nanooncology therapeutics. Nanooncology has produced refined methods of microsurgery, new molecular diagnostic techniques, earlier detection of tumors, and enhanced capabilities of the discovery of biomarkers for cancer. Molecular diagnostic improvements in brain cancer via nanomedicine may propel existing technologies to new heights of detection capability [5], aid in developing biomarkers in neuronal cancer cells with a higher affinity for more specific cell receptors in smaller sample volumes [6], and image solid tumors with a higher rate of 
specificity by utilizing quantum dots (QDs) and specific elemental nanoparticle forms [7]. Herein, we aim to discuss the future capabilities of nano-neurooncoloy by evaluating recent failures and triumphs in this exciting domain of medicine.

\section{Nanoparticle drug delivery across the blood brain barrier}

A specific area within which nanomedicine will have a tangible impact in neurooncology is through the provision of a novel method by which drugs, genes, and molecules can efficiently cross the blood brain barrier (BBB) with high kinematics and pharmocokinetics. Chemotherapy is conventionally administered orally or intravenously. Pharmacologically active chemoagents targeting the brain usually reach the tumor with low specificity and, more importantly, doselimiting toxicity. Oral administration exposes the anti-cancer drug to the metabolic system and could potentially cause unwanted pharmacokinetics, resulting in decreased tumor reactivity to the given dose. In parallel to this limitation, intravenously administered chemotherapy has low specificity which can be potentially harmful to nearby healthy tissue. Nanoparticle-based drug delivery may circumvent these common limitations by accessing the brain without exposing the anti-cancer drug to the metabolic system of the host organism. Further, nanoparticlechemoagent conjugates could potentially be directed to specifically accumulate within the tumor site, becoming active only within a specific region of the brain, thereby sparing healthy tissue from potential toxicities inherent with chemotherapeutic agents.

The control of elemental processes that occur at the molecular level are required in order to optimize the efficacy of oncology deliverables. The intravenous administration of drugs targeted to neuronal tumors must have the capacity to accurately locate the CNS upon introduction into the body, without causing systemic side-effects. Subsequently, they must traverse the BBB to exclusively target the tumor cells that they intend to destroy [8]. Ideally, the drug would be restricted to be active only in the regional vicinity of the target, at a specific point in time, and spare its influence on adjacent sensitive brain matter. Indiscriminate collateral damage could potentially be catastrophic in that the destruction of healthy brain tissue may interrupt essential neuronal cicuitry. Simultaneously, the potential benefits to a patient undergoing nanooncology treatment are significant. The challenge of achieving this ideal scenario includes optimizing elemental processes in engineering, physics, biology, chemistry, and pharmacology (Figure 2). Though these goals have not yet been achieved, a substantial amount of work toward the development of nanobiotechnology is propelling the biomedical community into an era that might realize the success of nanoparticle-enabled chemotherapeutic drug conjugates, which may efficaciously penetrate the BBB.

A

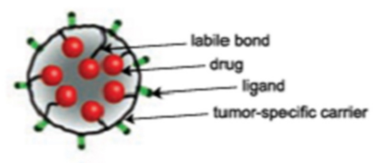

pro-drug: drug conjugated to
tumor-specific molecule

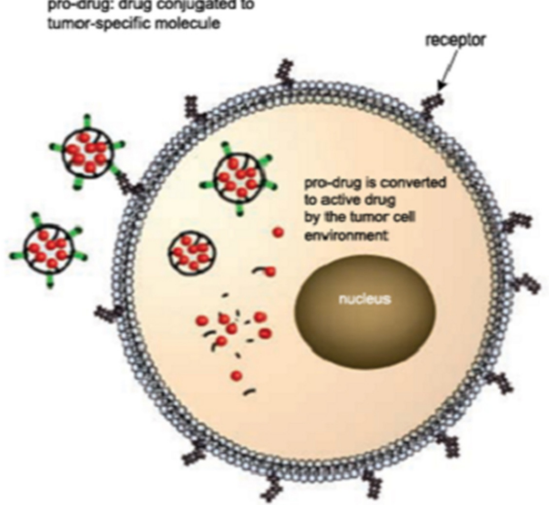

B

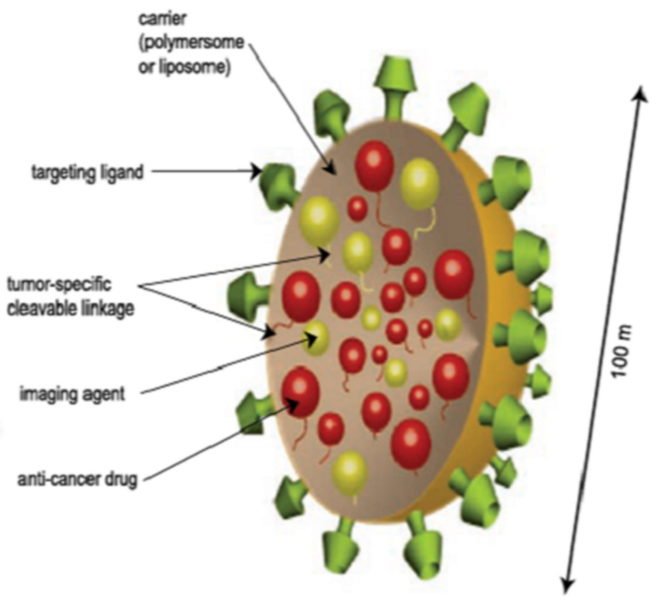




\section{FIGURE 2: Nanoparticle Applications}

Adapted with permission from Sinha, et al. [9] A: Tumor-activated drug delivery. B: Nanoparticles with both diagnostic and therapeutic functions.

In an attempt to deliver anti-tumoric drugs to a mouse model via nanoparticle technologies, Brigger, et al. utilized radiolabeled PEG-coated hexadecylcyanoacrylate nanospheres to target a gliosarcoma [10]. This study demonstrated a several-fold increase in the accumulation of the nanospheres within the targeted tumor, in comparison to surrounding brain tissue [10]. Garcia, et al. employed confocal microscopy to confirm that PEG-coated hexadecylcyanoacrylate nanoparticles can translocate successfully across the BBB via endocytosis in a safe mechanistic manner [11]. The translocation of nanoparticles across the BBB via endocytosis is not the only mechanism by which nanoparticles succeed at gaining entry to the brain. Silva, et al. states that "By conjugating different ligands to nanoparticle carriers, different transporters could potentially be recruited to facilitate the translocation of specific molecules or compounds. For example, nanoparticles composed of polyoxyethylene 21 stearyl ether conjugated with thiamine on their surfaces have been shown to cross the BBB via specific interactions with thiamine transporters" [8]. Feng, et al. developed PLGA nanoparticles containing the drug, paclitaxel, and administered them in 29 different types of cancer cell lines. In vitro experiments showed a 13-fold increase in cytotoxicity in all targeted cancers in comparison to those administered cancers being treated without the nanoparticle conjugate [12]. Of particular interest in this report is the observation that Feng, et al. were able to utilize different forms of spectroscopy to prove that paclitaxel was integrated into the nanoparticle sphere with high efficiency.

For the development of nanobiotechnologies in neurooncology, these techniques are important in order to ensure the pharmacokinetics of the drug within the brain. Further, Fang, et al. reported that the kinetics dictating the release of a therapeutic agent could be confidently and efficiently controlled [12]. The ability to develop specific nano-chemo agents that are under precise directives to release the drug within the tumor in an exclusive manner is imperative for successful treatment of future brain tumor patients in that it enables the clinicians to decrease the side-effects of the chemoagent by ensuring its administration only to the cancerous tissue within the brain. The development of these nano-sized medicinal elements is multifaceted in that it dictates a trajectory through basic science research, translational and clinical medicine, and industry partners (Figure 3). In a series of important experiments, Gulyaev, Kreuter and Steiniger, et al. demonstrated the successful attachment of the chemotherapeutic drug, doxorubicin, and neuropeptides to the surface of poly (butylcyanoacrylate) nanoparticles coated with polysorbate 80 [13-14]. Rodents treated with a doxorubicin polysorbate-coated nanoparticle showed higher survival rates than control groups. More than $20 \%$ of animals treated with the nanoparticle-doxorubicin conjugate showed long-term remission [15]. Histologically, tumor size in the treated group was smaller.

According to Silve, et al., the activity of polysorbate on the surfaces of the nanoparticles has two distinct effects on the nano-chemo conjugate: 1) it enables the incorporation of apolipoproteins B and E and 2) causes the nanoparticle to be taken up by brain capillary endothelial cells via receptor-mediated endocytosis [8]. The development of rat glioma models has allowed for the testing of these nanoparticle-chemoagent conjugates. Studies with these models have demonstrated significant remission with minimal toxicity [15]. These two properties are significant because although a significant amount of literature is dedicated to understanding how nanoparticles could cross the $\mathrm{BBB}$, a scarce amount is reported on the molecular and cellular mechanisms by which nanoparticles manage to cross the BBB in a safe manner. Understanding these physiological mechanisms is a prerequisite for creating safe 


\section{Cureus}

nanoparticle-based clinical trials in the future. To this note, poly (butylcyanoacrylate) nanoparticles coated with polysorbate 80 have been studied extensively and are now thought to be the first of a class of nanoparticles with a known cellular mechanism of crossing the BBB. Poly (butylcyanoacrylate) nanoparticles coated with polysorbate 80 contain apolipoproteins B and $\mathrm{E}$ which can naturally cross the BBB and subsequently become taken up by brain capillaries via receptor-mediated endocytosis [16-17]. The development of rodent models which can uptake nanoparticle-chemoagent conjugates and successfully transfer these agents across the BBB and attack the tumor while demonstrating minimal toxicity paves the way for future clinical trials.

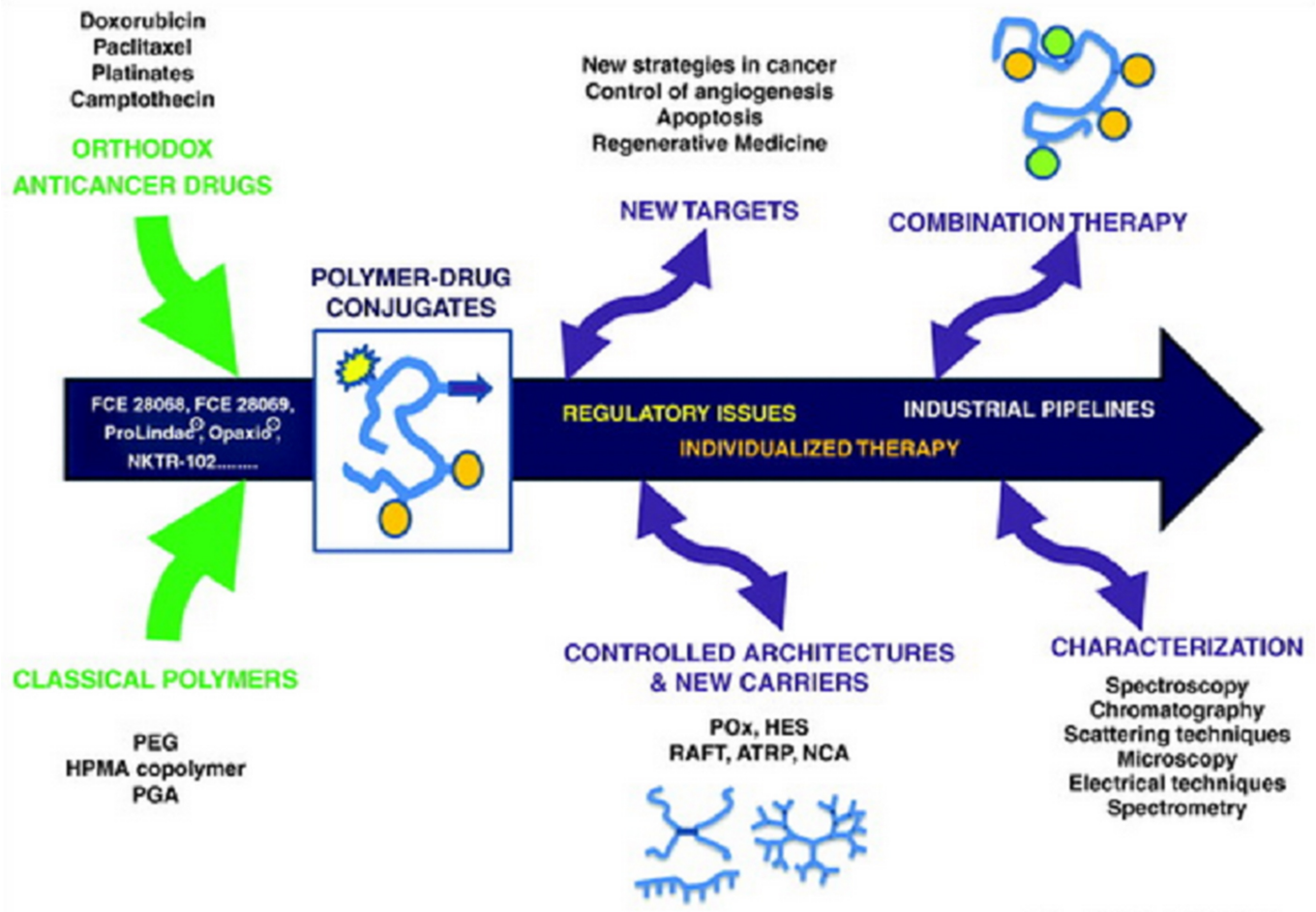

\section{FIGURE 3: Anticancer Nanoparticle Development}

"Polymer-drug conjugates as nano-sized medicines. Adapted from Canal, et al. [18] Reused with licensed permission: Current Opinion in Biotechnology (Elsevier license number: 3335641370510): Forefront nanobiotechnology with polymer-drug conjugate.

\section{Magnetic nanoparticles in neuro-oncology and thermo- ablation}

"Those diseases that medicines do not cure are cured by the knife. Those that the knife does not cure are cured by fire. Those that fire does not cure, must be considered incurable." -

Hippocrates, $370 \mathrm{BC}$ [19]. Glioblastoma is currently the most recalcitrant neurological cancer to treat. Although an initial resection of the tumor is possible, the recapitulation of the tumor, fueled by cancer stem cells, yields the ultimate demise of the patient. Residual infiltrating brain cancer cells are the target of a flurry of research initiatives aimed at decreasing the mortality rate in GBM patients. Particular nanobiotechnologies have emerged with potential capabilities to both diagnose and treat glioblastomas via an intrinsic heating mechanism deployed by the nanoparticle within the brain matter (Figure 4). The basics of this intracranial heating system are based on the peculiar paramagnetic properties of magnetic nanoparticles (MNPs) which allows for their detection by MRI imaging modalities [20-21]. The induction of hyperthermia to adjacent cells within the vicinity of the nanoparticle is a valuable asset of nanomedicine in 


\section{Cureus}

neurooncology. When exposed to alternating magnetic fields, MNPs are endowed with the capacity to induce hyperthermia [22]. The ramifications of temperatures of between 41 and 46 Celsius on cellular activity are significant: heat stress results in protein denaturation and abnormal folding, as well as DNA cross-linking within the nucleus, ultimately leading to apoptosis [23]. On the macro-level of the brain, hyperthermia induction via MNPs could potentially lead to altered $\mathrm{pH}$ states, perfusion, and oxygenation of the tumor microenvironment [24-25]. Increased oxygenation to the tumor microenvironment may lead to increased radiosensitivity in brain tumor treatment, as well as an increased efficacy of conventional brain tumor chemoagents [26-28].
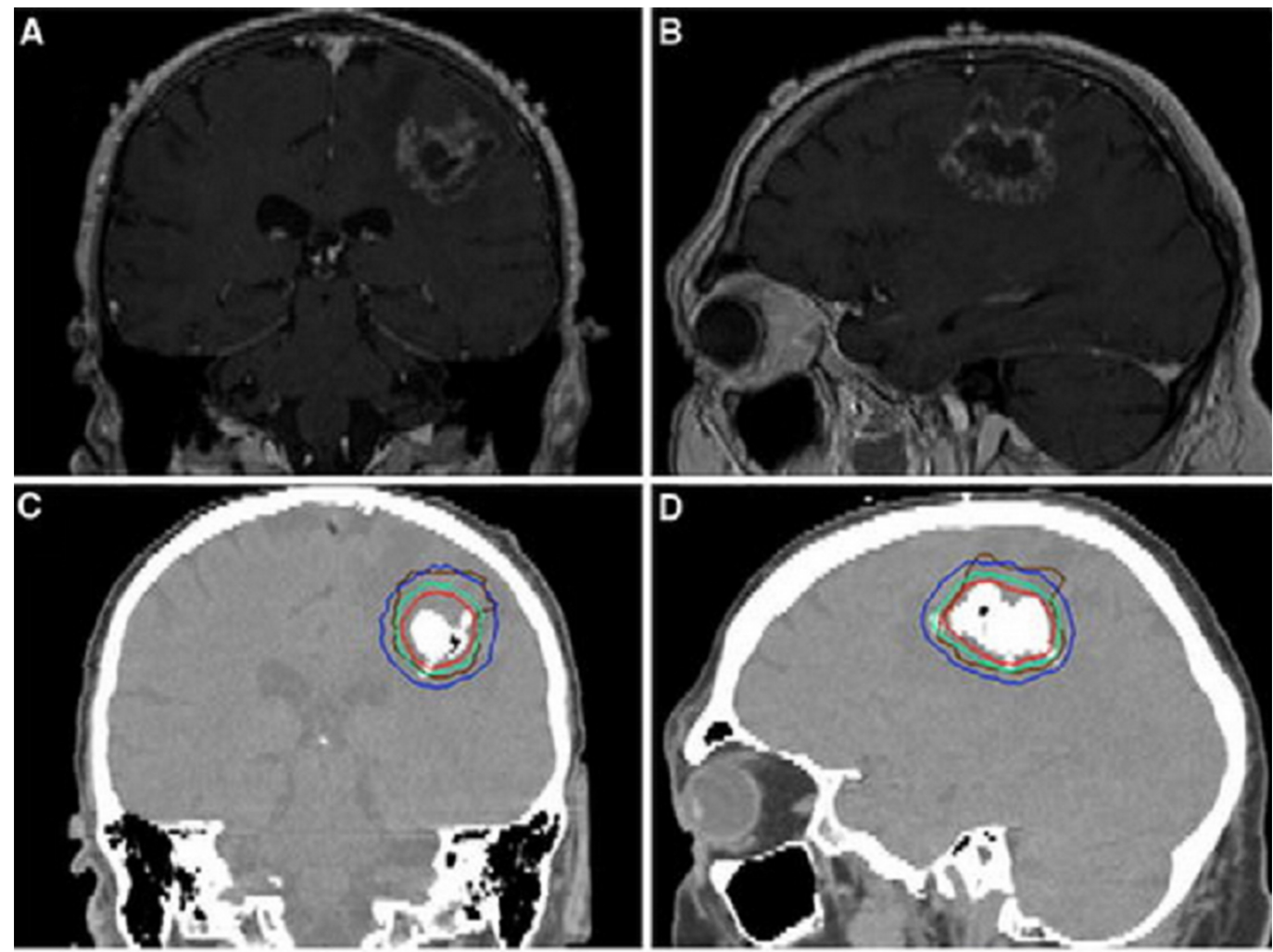

E

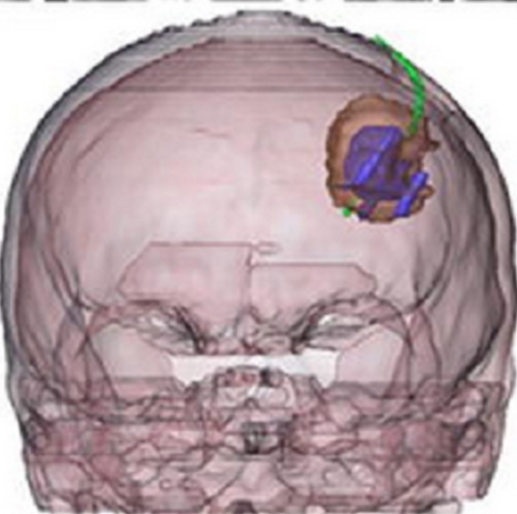

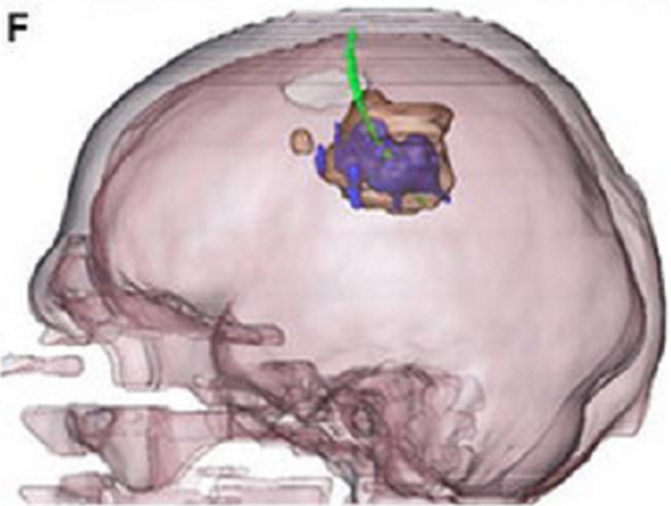

\section{FIGURE 4: Brain Tumor Imaging Illustrating Nanoparticle}

\section{Within GBM}

a,b: Pretreatment MRI of GBM. c,d: Hyperdense areas illustrating accumulation of magnetic nanoparticle deposits. Blue lines represent $40^{\circ} \mathrm{C}$ and red lines $50^{\circ} \mathrm{C}$. e,f: Reconstructed images using CT/MRI techniques portraying the magnetic fluid in blue, the tumor bulk in brown, and the thermometry catheter in green. Reused with licensed permission Springer. Credit to MaeirHauf, et al. Department of Neurosurgery, Bundeswehrkrankenhaus Berlin [29]. 


\section{Cureus}

The introduction of a specific alternating magnetic field (AMF) at the required amplitude and frequency to the MNPs induce hyperthermia by heating up the targeted nanoparticles [22]. Nduom, et al. analyzed the thermokinetics of the reactions which lead to thermal loss in the vicinity of brain tissue when MNPs are exposed to an AMF (Figure 5). MNPs convert magnetic energy into heat by means of dual synergetic mechanisms. As Nduom, et al. state, "Néel relaxation is caused by rapidly occurring changes in the direction of magnetic moments relative to crystal lattice. Brownian relaxation results from the physical rotation of MNPs within the medium in which they are placed. Both internal (Néel) and external (Brownian) sources of friction lead to a phase lag between applied magnetic field and the direction of magnetic moment, producing thermal losses" [22]. Thermotherapy via MNPs in brain tumors is a new avenue being explored by researchers worldwide to treat the most aggressive forms of brain cancer, GBM included. MNPs used for the induction of hyperthermia and eventual thermotherapeutic applications are engineered from a variety of elemental metals, including iron (Fe), manganese (Mn), cobalt (Co), zinc (Zn), and other oxides [30-34].

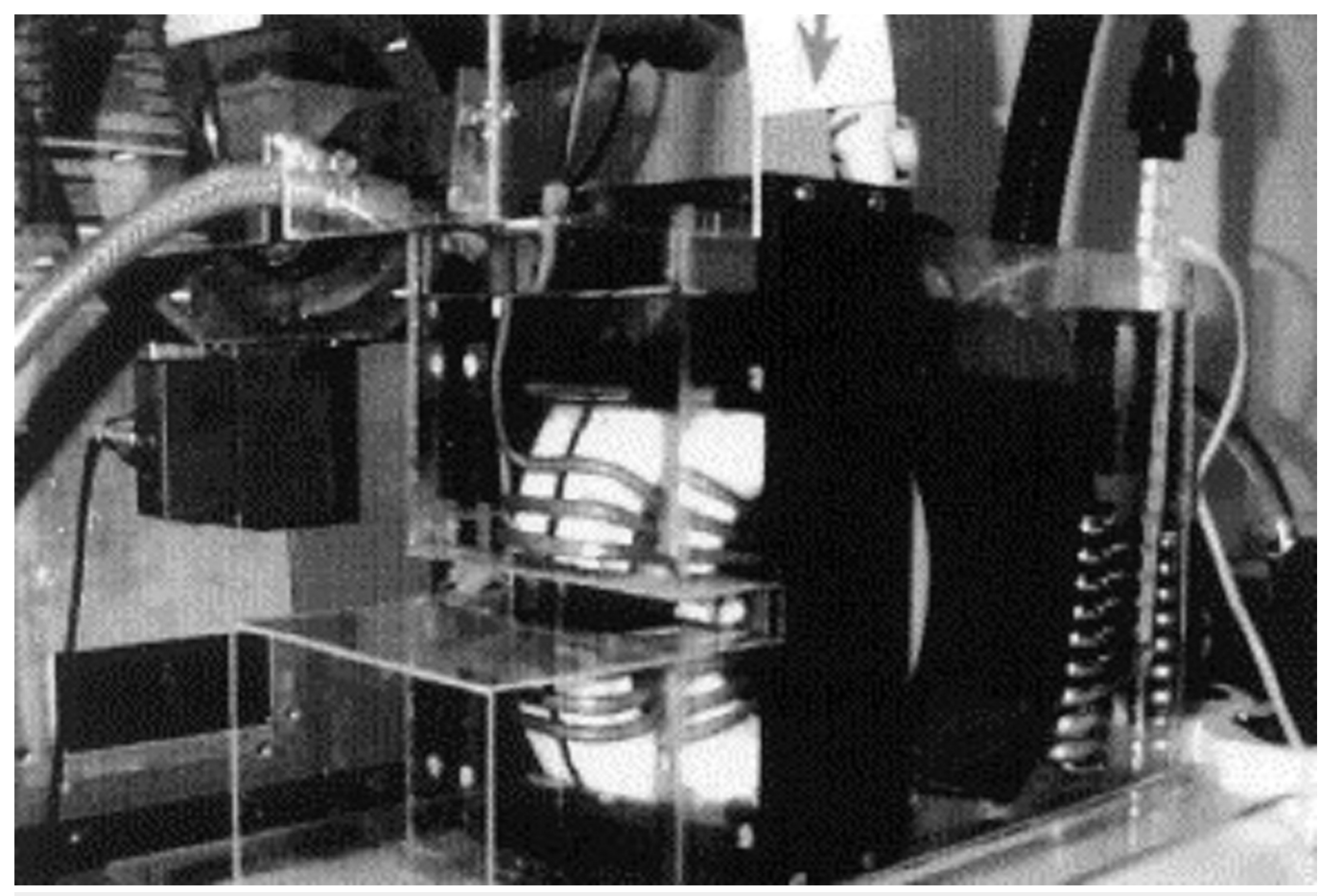

\section{FIGURE 5: Early Prototype of AMF Administration Device}

Engineered prototype of AMF administration at $500 \mathrm{kHz}$ and amplitudes of up to $10 \mathrm{kA} / \mathrm{m}$. Reused with permission fm. Elsevier (Image License Number: 3336861484008). Credit to Jordan A, et al. [35].

In 2006, Jordan, et al, demonstrated the positive efficacy of thermo-ablative mechanisms in GBM modeled rodents by employing aminosilane-coated iron-oxide nanoparticles [36]. Tumors were induced by implantation of RG-2-cells into the brains of 120 male Fisher rats. Tumor treatment was conducted using an AMF at a frequency of $100 \mathrm{kHz}$ and a variable field strength of $0-18 \mathrm{kA} / \mathrm{m}$. The survival time of the rodents involved in the study was used by Jordan, et al. to measure the efficacy of MNP thermotherapy. Hyperthermia induction with MNPs led to a 4.5 fold prolongation of survival relative to controls [36]. Hauf, et al. conducted an initial clinical trial using iron-oxide-based nanoparticles to induce thermo-ablation of glioblastomas in patients with recurrent tumors [37]. The human clinical trial by Hauf and colleagues injected brain tumors with aminosilane-coated iron-oxide nanoparticles and exposed the brain to an AMP of $100 \mathrm{kHz}$. The resulting thermal loss by the MNPs located within the brain tumor 


\section{Cureus}

demonstrated successful induction of hyperthermia within the brain tumor at a temperature of 51.2 degrees Celsius [37]. Patients did not experience side-effects, such as headache and nausea, and no neurological abnormalities or infections were detected in the treated region of the brain matter. Neuro-navigation techniques and thorough preoperative planning may be responsible for the lack of unwanted side-effects. In each patient, the navigation-guided puncture of the tumor was $100 \%$ successful, thereby preventing bleeding and inflammation. The median survival after recurrence was 13.4 months (95\% CI=10.6-16.2 months) and from the time of initial diagnosis was 23.2 months [37]. The success of this innovative clinical trial paves the way for safe and effective thermotherapy of cancerous brain tumors with the utilization of newly engineered MNPs (Figure 6). The overall results of this clinical trial demonstrated an increased survival rate in patients treated via thermotherapy and MNPs when compared to controls [37].

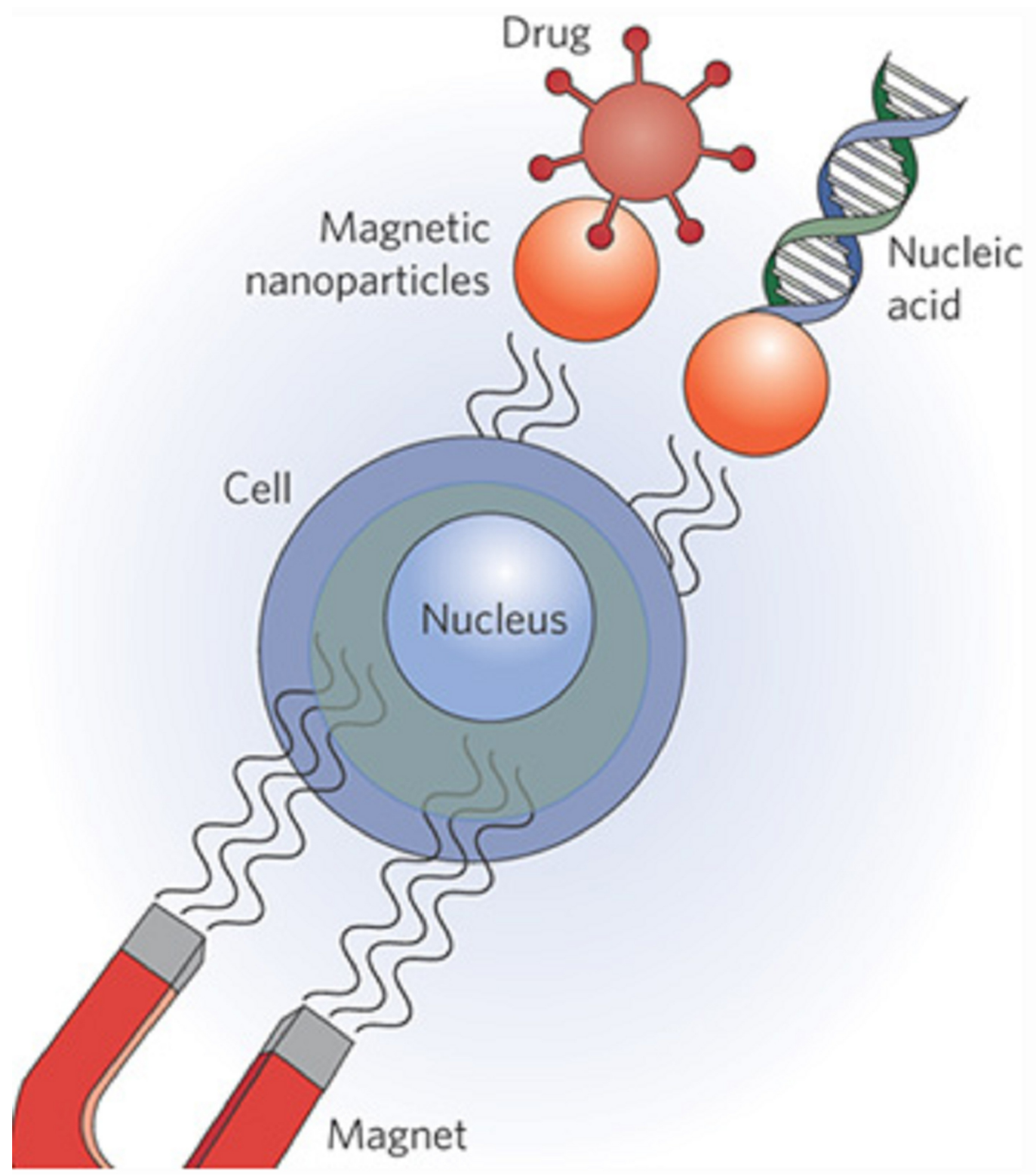

\section{FIGURE 6: Magnetic Nanoparticle Application}

Demonstration of potential applications of magnetic nanoparticles. Reused with licensed permission from Nature Nanotechnology (Image License number 3336880765668). Credited Plank, et al. [38]. 


\section{Conclusions}

In this review, we have presented findings to suggest that nanotechnology will serve as a viable candidate for potential treatments of various brain cancers, reducing both untoward morbidity and mortality from this devastating disease. Magnetic nanoparticles that can be MRIcompatible might provide the ideal theranostic platform for successful thermoablation, in addition to serving as a suitable candididate for precise drug delivery. We are encouraged, both from an industry and research perspective, by ongoing studies toward the safe use of nanomaterials for brain cancer treatment, and suggest that nanotechnology may serve as an ideal conduit the enhancement of the brain tumor therapeutic paradigm.

\section{Additional Information}

\section{Disclosures}

Conflicts of interest: In compliance with the ICMJE uniform disclosure form, all authors declare the following: Payment/services info: All authors have declared that no financial support was received from any organization for the submitted work. Financial relationships: All authors have declared that they have no financial relationships at present or within the previous three years with any organizations that might have an interest in the submitted work. Other relationships: All authors have declared that there are no other relationships or activities that could appear to have influenced the submitted work.

\section{Acknowledgements}

We would like to acknowledge Dr. Bruce Davidson, $\mathrm{PhD}$ and Claire Levine for their time to edit and give valuable suggestions to this manuscript.

\section{References}

1. Howlader N, Noone AM, Krapcho M, Garshell J, Neyman N, Altekruse SF, Kosary CL, Yu M, Ruhl J, Tatalovich Z, Cho H, Mariotto A, Lewis DR, Chen HS, Feuer EJ, Cronin KA (eds): SEER Cancer Statistics Review, 1975-2010. National Cancer Institute. 2013, http://seer.cancer.gov/csr/1975_2010/.

2. Invernici G, Cristini S, Alessandri G, et al.: Nanotechnology advances in brain tumors: The state of the art. Recent Pat Anticancer Drug Discov. 2011, 6:58-69.

3. Jain KK: Future prospects for the cure of brain cancer . Technol Cancer Res Treat. 2006, 5:1834.

4. Grosu AL, Weber WA, Franz M: Reirradiation of recurrent high-grade gliomas using amino acid PET (SPECT)/CT/MRI image fusion to determine gross tumor volume for stereotactic fractionated radiotherapy. Int J Radiat Oncol Biol Phys. 2005, 63:511-9.

5. Jain KK: Potential of nanobiotehnology in the management of glioblastoma multiforme . Glioblastoma: Molecular Mechanisms of Pathogenesis and Current Therapeutic Strategies. Ray SK (ed): Springer, New York; 2010. 399-419.

6. Jain KK: A Handbook of Nanomedicine. Humana/Springer, Totowa, NJ; 2008.

7. Jain KK: Advances in the field of nanooncology . BMC Med. 2010, 8:83.

8. Silva GA: Nanotechnology approaches for drug and small molecule delivery across the blood brain barrier. Surg Neurol . 2007, 67:113-116.

9. Sinha R, Kim GJ, Nie S, Shin DM: Nanotechnology in cancer therapeutics: Bioconjugated nanoparticles for drug delivery. Mol Cancer Ther. 2006, 5:1909-17.

10. Brigger I, Morizet J, Aubert G, et al.: Poly(ethylene glycol)-coated hexadecylcyanoacrylate nanospheres display a combined effect for brain tumor targeting. J Pharmacol Exp Ther. 2002, 303:928-36.

11. Garcia-Garcia E, Gil S, Andrieux K, et al.: A relevant in vitro rat model for the evaluation of blood-brain barrier translocation of nanoparticles. Cell Mol Life Sci. 2005, 62:1400-8.

12. Feng SS, Mu L, Win KY, et al.: Nanoparticles of biodegradable polymers for clinical administration of paclitaxel. Curr Med Chem. 2004, 11:413-24. 
13. Kreuter J, Alyautdin RN, Kharkevich DA, et al.: Passage of peptides through the blood-brain barrier with colloidal polymer particles (nanoparticles). Brain Res. 1995, 674:171-4.

14. Gulyaev AE, Gelperina SE, Skidan IN, et al.: Significant transport of doxorubicin into the brain with polysorbate-80 coated nanoparticles. Pharm Res. 1999, 16:1564-9.

15. Steiniger SC, Kreuter J, Khalansky AS, et al.: Chemotherapy of glioblastoma in rats using doxorubicin-loaded nanoparticles. Int J Cancer. 2004, 109:759-67.

16. Friese A, Seiller E, Quack G, et al.: Increase of the duration of the anticonvulsive activity of a novel NMDA receptor antagonist using poly(butylcyanoacrylate) nanoparticles as a parenteral controlled release system. Eur J Pharm Biopharm. 2000, 49:103-9.

17. Göppert TM, Müller RH: Plasma protein adsorption of Tween 80- and poloxamer 188stabilized solid lipid nanoparticles. J Drug Target. 2003, 11:225-31.

18. Canal F, Sanchis J, Vicent MJ: Polymer--drug conjugates as nano-sized medicines. Curr Opin Biotechnol. 2011, 22:894-900. 10.1016/j.copbio.2011.06.003

19. Jones S, Henry W: Hippocrates. Harvard University Press, Cambridge; 1959.

20. Sun C, Lee JS, Zhang M: Magnetic nanoparticles in MR imaging and drug delivery. Adv Drug Deliv Rev. 2008, 60:1252-65.

21. Jain TK, Richey J, Strand M, et al.: Magnetic nanoparticles with dual functional properties: drug delivery and magnetic resonance imaging. Biomaterials. 2008, 29:4012-21.

22. Nduom EK, Bouras A, Kaluzova M, et al.: Nanotechnology applications for glioblastoma. Neurosurg Clin N Am. 2012, 23:439-49. 10.1016/j.nec.2012.04.006

23. Goldstein LS, Dewhirst MW, Repacholi M, et al.: Summary, conclusions and recommendations: Adverse temperature levels in the human body. Int J Hyperthermia. 2003, 19:373-84.

24. Hildebrandt B, Wust P, Ahlers O, et al.: The cellular and molecular basis of hyperthermia . Crit Rev Oncol Hematol. 2002, 43:33-56.

25. Wust P, Hildebrandt B, Sreenivasa G, et al.: Hyperthermia in combined treatment of cancer . Lancet Oncol. 2002, 3:487-497.

26. Vanpouille-Box C, Lacoeuille F, Belloche C, et al.: Tumor eradication in rat glioma and bypass of immunosuppressive barriers using internal radiation with (188)Re-lipid nanocapsules. Biomaterials. 2011, 32:6781-90. 10.1016/j.biomaterials.2011.05.067

27. Hirsch LR, Gobin AM, Lowery AR, et al.: Metal nanoshells. Ann Biomed Eng. 2006, 34:15-22.

28. Bernardi RJ, Lowery AR, Thompson PA, et al.: Immunonanoshells for targeted photothermal ablation in medulloblastoma and glioma: an in vitro evaluation using human cell lines. J Neurooncol. 2008, 86:165-72.

29. Maier-Hauff K, Ulrich F, Nestler D, et al.: Efficacy and safety of intratumoral thermotherapy using magnetic iron-oxide nanoparticles combined with external beam radiotherapy on patients with recurrent glioblastoma multiforme. J Neurooncol. 2011, 103:317-24.

30. Lee JH, Jang JT, Choi JS, et al.: Exchange-coupled magnetic nanoparticles for efficient heat induction. Nature Nanotechnology. 2011, 6:418-22.

31. Sharma R, Chen CJ: Newer nanoparticles in hyperthermia treatment and thermometry . J Nanoparticle Research. 2009, 11:671-689.

32. Pradhan P, Giri J, Samanta G, et al.: Comparative evaluation of heating ability and biocompatibility of different ferrite-based magnetic fluids for hyperthermia application. J Biomed Mater Res B Appl Biomater. 2007, 81:12-22.

33. Atsarkin VA, Levkin LV, Posvyanskiy VS, et al.: Solution to the bioheat equation for hyperthermia with $\mathrm{La}(1-\mathrm{x}) \mathrm{Ag}(\mathrm{y}) \mathrm{MnO}$ (3-delta) nanoparticles: the effect of temperature autostabilization. Int J Hyperthermia. 2009, 25:240-7. 10.1080/02656730802713565

34. Bae S, Lee SW, Takemura Y, et al.: Dependence of frequency and magnetic field on selfheating characteristics of NiFe2O4 nanoparticles for hyperthermia. IEEE Transactions on Magnetics. 2006, 42:3566-3568.

35. Jordan A, Scholz R, Wust P, et al.: Magnetic fluid hyperthermia (MFH): Cancer treatment with AC magnetic field induced excitation of biocompatible superparamagnetic nanoparticles. J. Mag and Mag Mater. 1999, 201:413-419.

36. Jordan A, Scholz R, Maier-Hauff K, et al.: The effect of thermotherapy using magnetic nanoparticles on rat malignant glioma. J Neurooncol. 2006, 78:7-14.

37. Maier-Hauff K, Rothe R, Scholz R, et al.: Intracranial thermotherapy using magnetic nanoparticles combined with external beam radiotherapy: results of a feasibility study on patients with glioblastoma multiforme. J Neurooncol. 2007, 81:53-60. 


\section{Cureus}

38. Plank C: Nanomedicine: Silence the target. Nature Nanotechnology. 2009, 4:544 - 545. 